\section{Removing Halftone Patterns From Scanned Images}

John Russ ${ }^{1}$ and Chris Russ ${ }^{2}$

1. North Carolina State University, John_Russ@NCSU.edu

2. Reindeer Games, Inc., jcr6@AOL.com

Using illustrations from published technical articles is part of many lectures and presentation citations, and given the availability of flat bed scanners, should be easy to accomplish. The problem that remains is removing halftone patterns and other periodic noise that result from printing and scanning technology. Practically all magazines and newspapers are printed using a regular halftone pattern that uses an array of dots varied in size to produce the visual illusion of continuous gray scale. Color images are usually printed with three, four or even more such patterns using different colored inks and different pattern orientations. Scanning such images into a computer can introduce a further pattern due to the moiré interference between the printed pattern and the spacing of the sensors in the scanner. Such patterns are also characteristic of images obtained from single-chip video cameras because of the color filters present in front of the light sensors on the chip.

Elimination of these patterns is necessary as a precursor to most serious use of the images. The most common and typical recommendation for doing so is to use various degrees of smoothing or blurring to fill in the gaps between the dots, but this inevitably results in the loss of a great deal of sharpness of the features that are of interest and can erase small features and narrow lines entirely. Blurring is not a good solution. Other recommendations that sometimes appear in internet discussion groups are to rotate the image and rescan it, trying to find a chance orientation that will minimize the moiré pattern. This rarely produces satisfactory results. Some scanners contain built-in filters to reduce such patterns, but usually at the cost of image sharpness.

There is a very straightforward way to correct this type of periodic noise in images. It requires a few tools for image processing and a little understanding of what is going on. While the

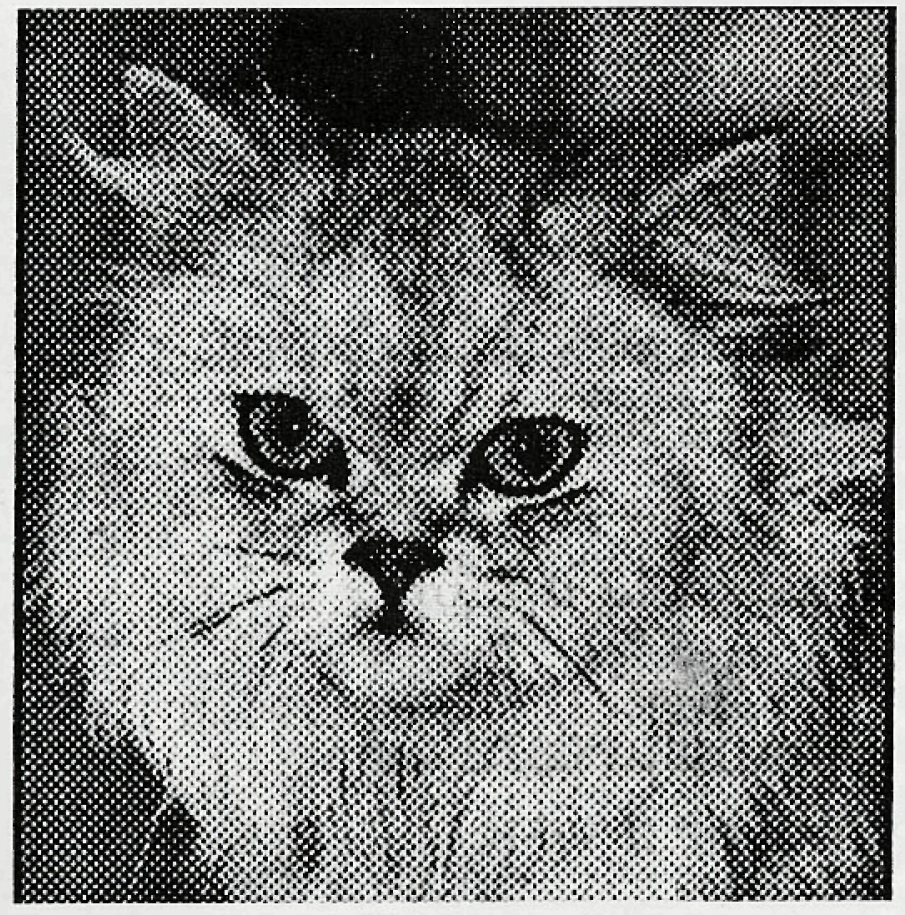

Figure 1. Original scanned image technique utilizes Fourier transforms and works in frequency space instead of the original array of pixels in the image, there is nothing in it that requires any mathematics knowledge or programming skills on the part of the user. The whole operation is quite interactive and fast, and can become quite intuitive with a little practice.

Many image processing programs can perform Fourier transforms on images, including free ones such as $\mathrm{NIH}$ Image. In the example shown here, the Image Processing Tool Kit was used. It includes a comprehensive set of tools for working in Fourier or frequency space. The Tool Kit can be used with many programs (both Mac and Windows based) that accept Photoshop-compatible plugins. The same operations shown below can be very effectively performed using NIH-Image for grey scale images. With either Photoshop or $\mathrm{NIH}$-Image, a macro or action can be constructed to perform the various steps automatically.

Figure 1 shows an example of an image scanned in from a newspaper. The halftone dots are very evident. This is actually a small portion ( $512 \times 512$ pixels square) from the original image, enlarged to show the halftone dots. Figures 4 and 7 show the same region after processing. The fast Fourier transform (FFT) used requires that images have dimensions that are exact powers of 2. There are several ways to deal with images of arbitrary dimension, the most common one being to pad the image up to the next larger power-of-two size by pasting it into a larger canvas and filling the outside with the mean grey scale value of the image.

Figure 2 shows the power spectrum from a Fourier transform. In the power spectrum, the magnitude of low frequency information (gradually varying brightness as a function of position in the original image) is plotted in the center of the array and high frequency information (rapidly varying brightness with position) is plotted at increasing radii outwards. The pixel values (darkness) represent the logarithm of the amplitude of the various sinusoidal components in the image. The dark spots ("spikes") in the power spectrum far away from the center are an indication of periodic noise in the image. They align with the repetitive pattern of dots, and their darkness indicates how much of various frequencies are present. Removing them is equivalent to removing the periodic noise.

Figure 3 shows a mask that was produced to remove these spikes. There are quite a few ways to produce such a mask, including manually with the Photoshop paint brush. The Tool Kit also includes several automatic methods including a Top Hat filter. This is a filter that finds spikes regardless of the local background level and allows thresholding to remove the rest of the image. The black

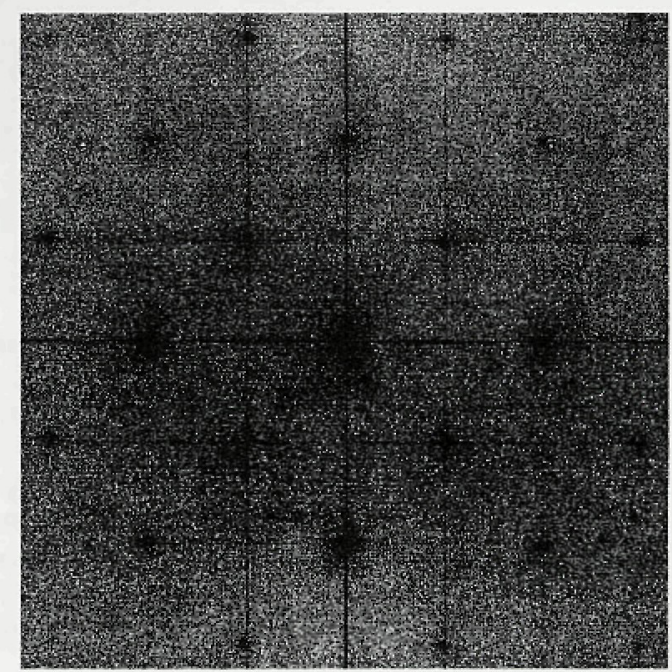

Figure 2. Power spectrum from original image, showing spikes due to halftone pattern 


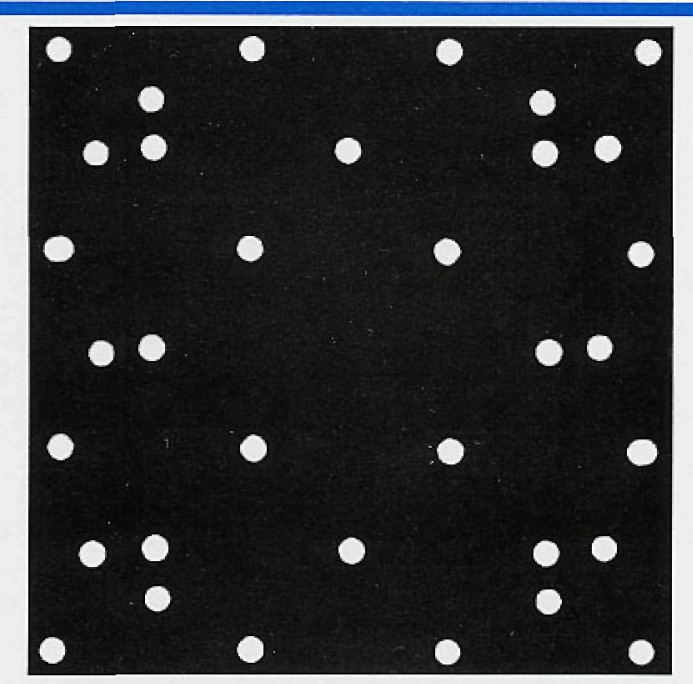

Figure 3. Mask used to remove spikes from power spectrum in Figure 2.

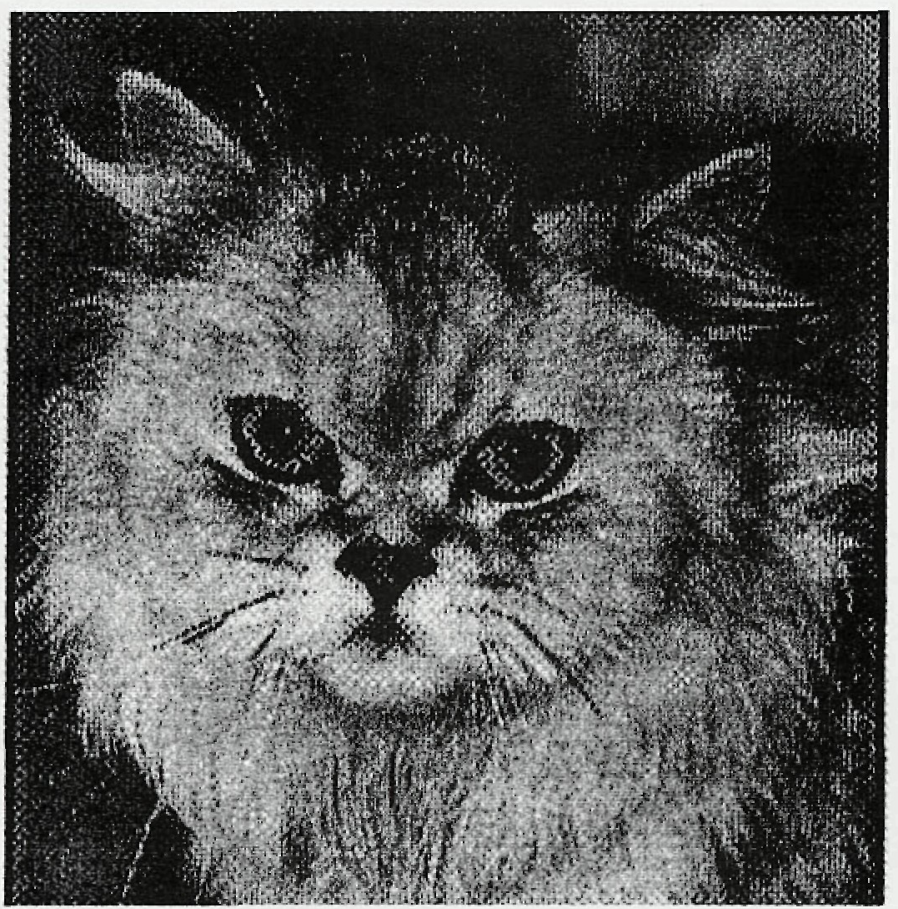

Figure 4, Image reconstructed after removal of spikes using mask in Figure 3.

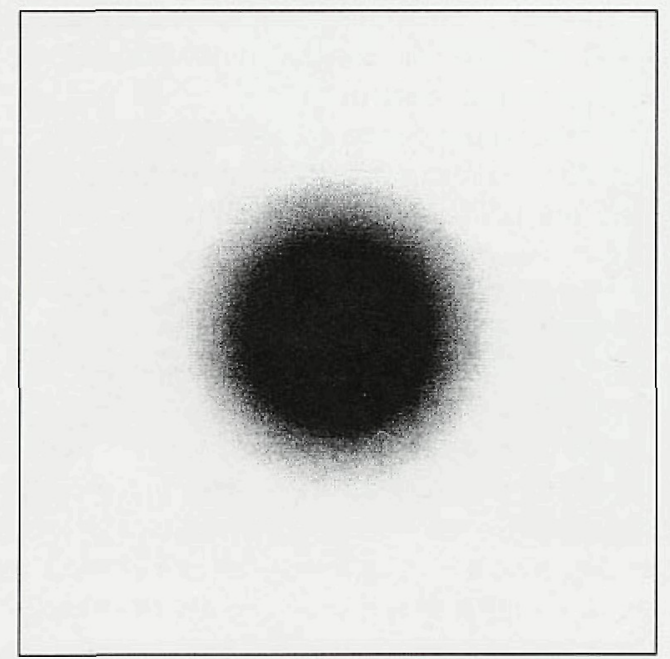

Figure 5. Butterworth filter used to reduce amplitude of high frequency terms

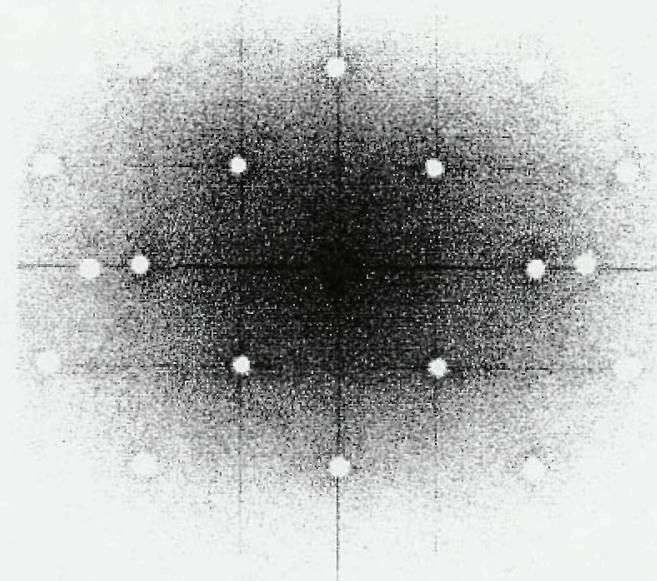

Figure 6. Power spectrum after application of both filters

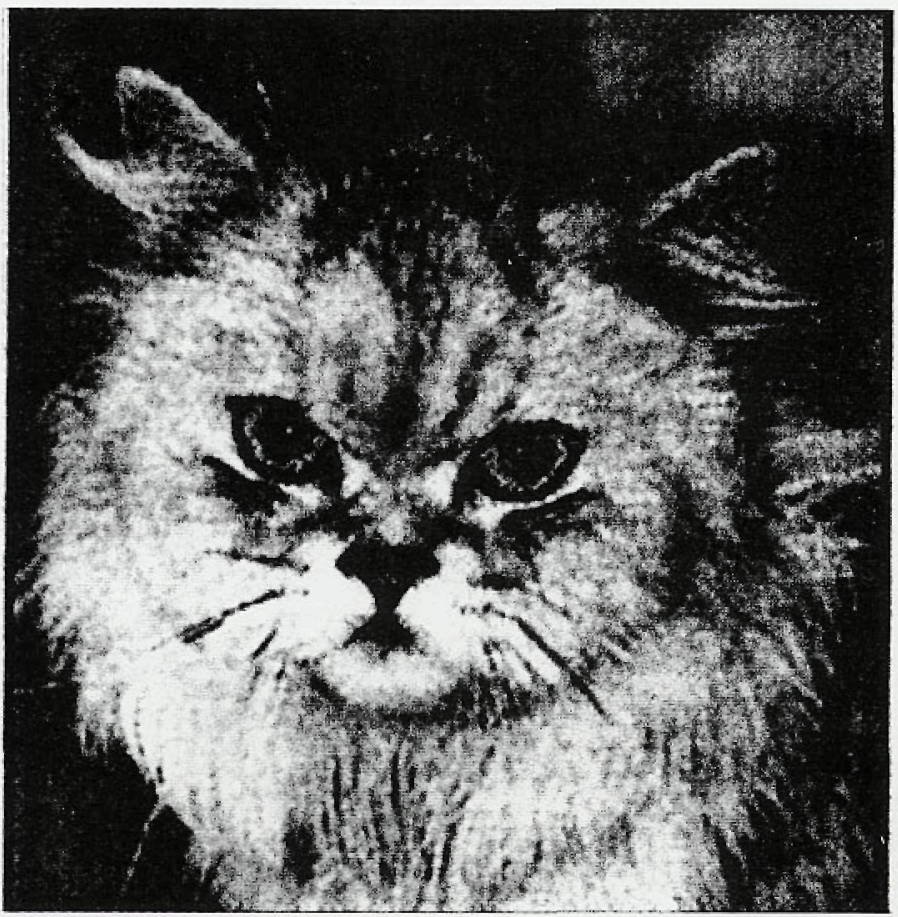

Figure 7. Resulting image

values in the mask keep the full value of all magnitudes associated with the desired information in the image, while the white spots will erase the spikes. A small Gaussian blur has been applied to the mask so that the pixels on the edges of the holes in the mask are set to grey, which improves the results.

Figure 4 shows the result of applying this mask as a filter to remove the spikes. The periodic noise has been removed without affecting any of the other information present. There is still some pattern evident because the image has been scanned at a higher magnification than it was printed, and the halftone dots are spaced farther apart than their size (which is typical of newsprint halftones).

Figure 5 shows a second filter that was employed to correct the remaining noise. This is a Butterworth second-order high-frequency cut-off filter (the Tool Kit has selections to create a variety of high- and low-pass filters), which keeps low frequencies (gradual variations in grey scale) while progressively cutting off higher ones (the more rapid variations associ-

Continued on following page 


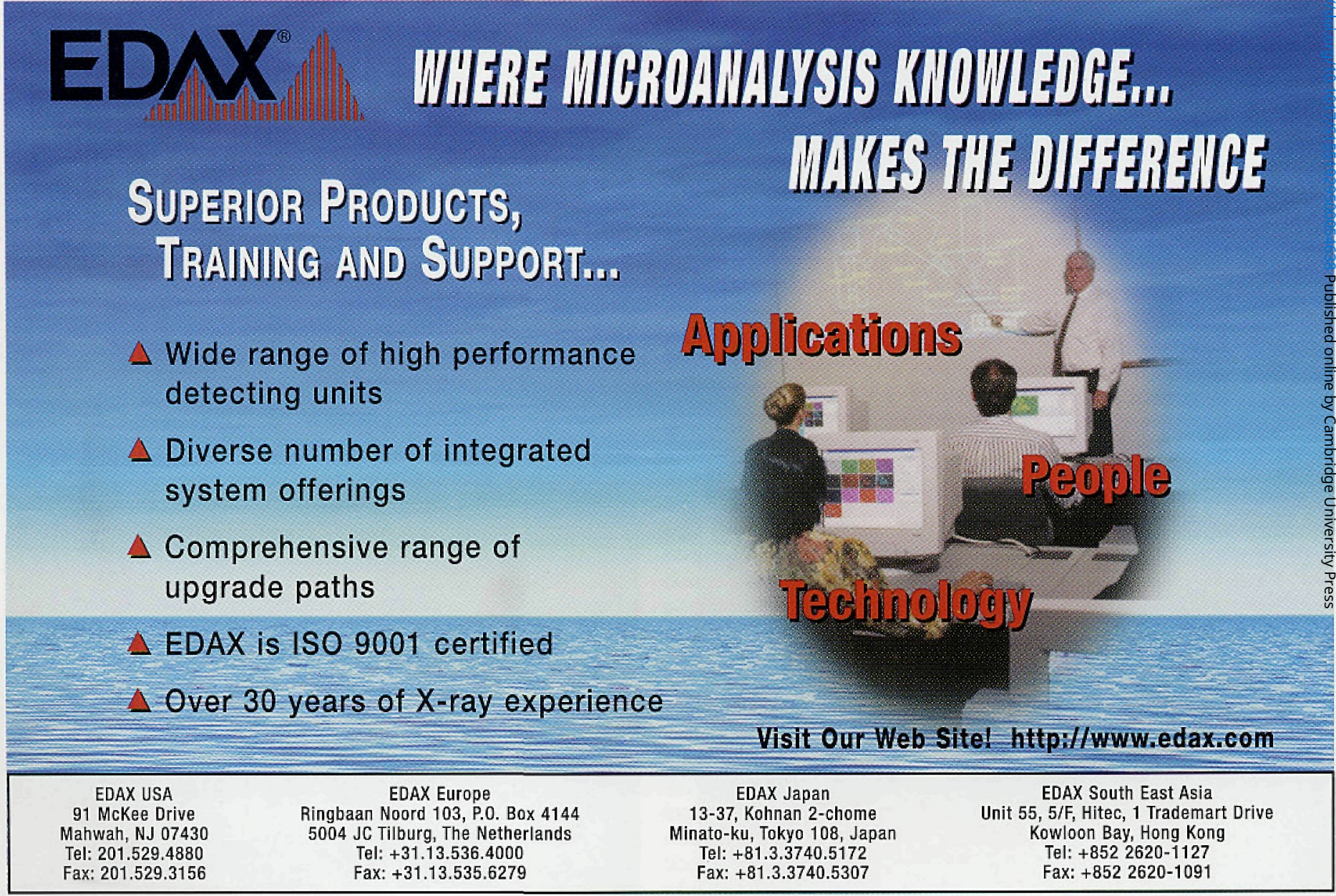

\section{Removing Halftone Patterns From Scanner Images Continued from Preceding Page}

ated with edges of the printed dots). In this case the mid-point of the cutoff was set to the spacing of the half-tone dots in the original image.

Figure 6 shows the final version of the power spectrum, with the periodic spots removed and the high frequencies attenuated but not eliminated. It is generally a useful learning tool to examine the power spectrum as you apply various masks and filters. Modifying the image in Fourier space makes it quite easy to remove periodic noise (or in some cases to keep periodic information and remove random noise) and to tailor the frequency response of the resulting image in ways that are difficult or impossible when working with the original pixel array.

Figure 7 shows the result of applying both filters. Now in addition to the removal of the periodic noise, the random pixel variations are also reduced, the image sharpness and contrast have been restored, and all of the details that are present in the image can be clearly seen. Note that even the cat's whiskers which are barely discernible in the original image can be clearly seen. The use of a smoothing or blur function would have erased these fine lines long before the halftone dots were smoothed together.

For a color image, in which the various halftone patterns for each ink have different orientations, the procedure would be to separate the image into color planes or channels corresponding to each color (CMYK for printed images, RGB for scanner or camera artifacts). Each image is then treated separately using the same operations as above, after which the processed color planes are recombined to produce an enhanced color image.

\section{Microwave Immunohistochemistry on Mohs Cryostat Sections}

Diane G. Miller, Miller Consultant Service millerd@coho.net

With the advent of Mohs surgery in the dermatology clinics, making sure the margins are clear of any cancer is of the utmost importance to both patient and dermatopathologist. While the patient is still in the surgery suite, immunohistochemstry staining can be done in the laboratory microwave (Pelco microwave with water load cooler, wattage controller and temperature probe). The immunohistochemistry procedure takes only 25-30 minutes as compared to the more conventional methods that take 2 hours or more.

The microwave needs to be checked for hot spots using the Pelco \#36140 microwave bulb array. Make sure the area in which you are going to put your slides has no hot spots, indicated by no illuminating bulbs. Using a Sigma Diagnostics \#H6644 Humid Chamber, place a paper towel in the bottom of the chamber and fill with $1 / 4$ inch of water. This is in addition to the water loads used to eliminate hot spots. Cut a very small hole into the top of the humid chamber-just large enough for the probe to fit into-at the exact point that the probe will be immersed in the reagent that is covering the tissue section. The temperature probe will measure the reagent temperature on the slide, maintaining a constant temperature of $40^{\circ} \mathrm{C}$. You can run up to six slides at once in a single humid chamber.

Vaporization is very important to this procedure. The vapor is contained in the humid chamber and enhances the reaction. Reagents used are the Vector Universal Elite Kit, Vector DAB kit 